\title{
Review
}

\section{Trends and Opportunities in Tick-Borne Disease Geography}

\author{
Catherine A. Lippi, ${ }^{1,2,8, \bullet}$ Sadie J. Ryan,,$^{1,2,3, \bullet}$ Alexis L. White, ${ }^{1,2, \bullet}$ Holly D. Gaff, ${ }^{4,5, \bullet}$ and \\ Colin J. Carlson ${ }^{6,7}$
}

'Quantitative Disease Ecology and Conservation (ODEC) Lab, Department of Geography, University of Florida, Gainesville, FL 32601, USA, ${ }^{2}$ Emerging Pathogens Institute, University of Florida, Gainesville, FL 32611, USA, ${ }^{3}$ College of Life Sciences, University of KwaZulu Natal, Durban, 4041, South Africa, ${ }^{4}$ Department of Biology, Old Dominion University, Norfolk, VA 23529, USA, ${ }^{5}$ School of Mathematics, Statistics and Computer Science, University of Kwa-Zulu Natal, Durban, 4041, South Africa, ${ }^{6}$ Center for Global Health Science and Security, Georgetown University Medical Center, Georgetown University, Washington, DC, 20007, USA, 'Department of Microbiology and Immunology, Georgetown University Medical Center, Georgetown University, Washington, DC, 20007, USA, and ${ }^{8}$ Corresponding author, e-mail: clippi@ufl.edu

Subject Editor: Stephen Rich

Received 28 January 2021; Editorial decision 15 April 2021

\begin{abstract}
Tick-borne diseases are a growing problem in many parts of the world, and their surveillance and control touch on challenging issues in medical entomology, agricultural health, veterinary medicine, and biosecurity. Spatial approaches can be used to synthesize the data generated by integrative One Health surveillance systems, and help stakeholders, managers, and medical geographers understand the current and future distribution of risk. Here, we performed a systematic review of over 8,000 studies and identified a total of 303 scientific publications that map tick-borne diseases using data on vectors, pathogens, and hosts (including wildlife, livestock, and human cases). We find that the field is growing rapidly, with the major Ixodes-borne diseases (Lyme disease and tick-borne encephalitis in particular) giving way to monitoring efforts that encompass a broader range of threats. We find a tremendous diversity of methods used to map tick-borne disease, but also find major gaps: data on the enzootic cycle of tick-borne pathogens is severely underutilized, and mapping efforts are mostly limited to Europe and North America. We suggest that future work can readily apply available methods to track the distributions of tick-borne diseases in Africa and Asia, following a One Health approach that combines medical and veterinary surveillance for maximum impact.
\end{abstract}

Key words: tick-borne diseases, maps, geospatial, prevalence species distribution modeling

Tick-borne diseases are increasingly recognized as a neglected subset of emerging infections. The expansion of Lyme disease (Lyme borreliosis) in the United States and Europe has brought attention to the ecological dimensions of their emergence, and the broader links between global change and the expansion and resurgence of vector-borne disease. More recently, in the United States, the spread of Powassan virus and spotted fever group rickettsioses have been seen as evidence of an emerging trend: tick-borne pathogens are proliferating, spreading to new areas, and emerging in human populations, at a comparable rate to other zoonotic threats (Woolhouse et al. 2008, Smith et al. 2014). These perspectives, of course, focus predominantly on Western countries, where zoonotic diseases have a comparatively lower burden (Torgerson and Macpherson 2011, Kuris 2012).
Worldwide, tick-borne diseases are a persistent example of problems at the One Health interface between humans, wildlife, and agriculture. Many, like Crimean-Congo hemorrhagic fever (CCHF) and tick-borne encephalitis, are a particularly significant problem for impoverished livestock keepers in rural locations (Grace et al. 2017, Espinaze et al. 2018). These conditions are severely neglected, often receiving less clinical attention and dedicated public health funding than directly-transmitted zoonotic viruses like influenza (Tick-Borne Disease Working Group 2018). Ticks are usually prioritized below mosquitoes by vector control programs, given the comparatively lower global public health burden and often limited agency resources. Vector control to reduce tick populations is further limited by the availability of large-scale mitigation strategies and control technologies, where additional research may be required to develop effective 
control measures for ticks and wildlife hosts (White and Gaff 2018, Rochlin et al. 2019, Eisen and Stafford 2020). Prevention and treatment in clinical settings are similarly limited: despite their frequently severe prognosis and high case fatality rate, few tick-borne pathogens have available or widely used vaccines, and only one research laboratory in the world regularly works with tick-borne pathogens at BSL4 containment. Detection and diagnosis of many tick-borne infections in humans are also challenging, given the broad clinical presentation of many tick-borne diseases, the current availability of reliable diagnostic tests, and multi-tiered approaches needed to confirm pathogens (Fatmi et al. 2017, Bush and Vazquez-Pertejo 2018).

The relative neglect of tick-borne illnesses among vector-borne diseases is also evident in basic disease surveillance data. Lyme surveillance is well established, and several large clinical datasets have been curated and used by researchers; and a handful of tick-borne zoonoses, such as CCHF, are notifiable in systems like ProMed-mail and the WHO Disease Outbreak News (ProMED 2021, WHO 2021). But on the whole, tick-borne pathogens are severely under surveilled, meaning many outbreaks likely go unreported, and the distributions and burdens of these diseases are likely underestimated or entirely unknown (Schiffman et al. 2016, Eisen and Paddock 2020). In no small part, this reflects the unique challenges of acquiring and verifying data on tick-borne diseases for spatial analyses. The vectors and within-vector pathogens may have distinct distributions, and human case data may also differ in its distribution, as a function of encounter and exposure (Brown et al. 2005, Lippi et al. 2020). In addition, vector and human case data require different methods of data collection, each with logistical constraints that may dictate the geographic extent of sampling. Given these challenges, tick distribution maps are often used as a proxy for either transmission exposure risk, or to describe the human disease distribution, but vector range is poorly characterized for most tickborne pathogens. Moreover, nearly all tick-borne infections of humans are zoonotic, and many have wildlife hosts, where data on infection in each layer of human, domesticated, and wildlife host, describe different components of the transmission process (Brown et al. 2005). Data on animal reservoirs are not always collected by existing health surveillance networks and are rarely stored in the same geoinformatic systems.

Maps are a primary tool for visualizing spatial information regarding pathogens and communicating the potential risk of exposure. Disease maps have long been used in public health to describe the distribution of vector-borne diseases, ranging in complexity from plotted cases (i.e., dot maps) to projected risk predictions modeled with machine learning algorithms (Kitron 1998, Carlson 2020). Regardless of complexity, mapped products rely on the availability of georeferenced datasets. Given the challenges surrounding tick-borne disease research, we hypothesized that most tick-borne diseases have not been comprehensively mapped. To evaluate the state of the field, we performed a systematic literature review and identified all studies of tick-borne pathogens that produced spatial data, models, or other mapping analyses of the pathogens themselves, or used maps of the vectors as a proxy. We found that despite the obvious threat posed to human and animal health by these diseases-and their growing significance in a changing world-the vast majority are undermapped, and many pathogens have not been mapped at all. Based on our results, we identify trends in the field, including shifting priorities for surveillance and methodological innovation, and discuss where surveillance efforts may need to be supplemented in the coming years.

\section{Methods}

We compiled a list of twenty-seven tick-borne pathogens of medical concern for inclusion in literature searches, using data from (Dantas-Torres et al. 2012) and (Brackney and Armstrong 2016). Four additional pathogens of recent public health interest were also included for review: Borrelia mayonii (Pritt et al.; Spirocheatales: Spirochaetaceae), B. miyamotoi (Fukunaga et al.; Spirocheatales: Spirochaetaceae), Rickettsia parkeri (Lackman et al.; Rickettsiales: Rickettsiaceae) (CDC 2018), and Panola mountain ehrlichiosis (Ehrlichia spp.) (Reeves et al. 2008). A final list of pathogens and vectors included in the study is available in Tables 1 and 2 .

We conducted literature searches following Preferred Reporting Items for Systematic Reviews and Meta-analyses (PRISMA) statement guidelines, a checklist of criteria to ensure transparency in systematic reviews (Liberati et al. 2009, Moher 2009). Searches for each pathogen, and named diseases they cause, were conducted in the PubMed Central (PMC) and Google Scholar databases from January to September 2020. The search queries used included combinations of pathogen names and key terms used to describe mapping and spatial analysis studies, taking the format: [species name] OR [disease name] AND ("SaTScan" OR "MaxEnt" OR "spatial cluster" "OR "spatial analysis" OR "geospatial" or "ecological niche model*" OR "mapping" OR "nearest neighbor" OR "spatial GLM*" OR "species distribution model" "). We did not place restrictions on the geographic region of study or date of publication, and searches were limited to English language results. Additional novel records for screening were taken from cited literature in records identified via database searches.

Duplicate records were removed from search results, and the remaining papers were screened for further review. Original,

Table 1. Viruses included in the study

\begin{tabular}{|c|c|c|}
\hline Pathogen & Family & Vectors \\
\hline African swine fever virus & Asfarviridae & Ornithodoros spp. \\
\hline bourbon virus & Orthomyxoviridae & Amblyomma spp. \\
\hline Colorado tick fever virus & Reoviridae & Not in literature \\
\hline Crimean-Congo hemorrhagic fever virus & Bunyaviridae & $\begin{array}{l}\text { Hyalomma spp. } \\
\text { Rhipicephalus spp. }\end{array}$ \\
\hline Heartland virus & Bunyaviridae & $\begin{array}{l}\text { Amblyomma spp. } \\
\text { Haemaphysalis spp. }\end{array}$ \\
\hline Huaiyangshan banyangvirus & Bunyaviridae & Haemaphysalis spp. \\
\hline Kyasanur forest disease virus & Flaviviridae & Haemaphysalis spp. \\
\hline Louping Ill virus & Flaviviridae & Ixodes spp. \\
\hline Nairobi sheep disease virus & Bunyaviridae & Haemaphysalis spp. \\
\hline Omsk hemorrhagic fever virus & Flaviviridae & Not in literature \\
\hline Powassan virus & Flaviviridae & Ixodes spp. \\
\hline Sawgrass virus & Rhabdoviridae & Not in literature \\
\hline Tick-borne encephalitis virus & Flaviviridae & $\begin{array}{l}\text { Dermacentor spp. } \\
\text { Ixodes spp. }\end{array}$ \\
\hline
\end{tabular}


peer-reviewed studies with a spatial component, and within the taxonomic scope of the review, were assessed for further screening. The remaining full-text studies were reviewed for inclusion. Literature reviews, expert commentaries, synthesis papers, conference abstracts, and unpublished theses were excluded from results, as were studies using serology not resolved to the taxonomic level of interest for a given pathogen, and studies that focused on pathogens solely known for their veterinary importance. We recorded the citation, DOI link, geographic region, pathogens, vectors, data sources and sampling methods for vectors, and data inputs for each study included in our final dataset.

Mapping and spatial analysis methods were also recorded for papers. In order to describe the types of maps in the studies, we created a key, based on a previous study of helminth parasite mapping (Schluth et al. 2020), and classified studies into eight types. Studies could contain more than one type of map (Table 3).

\section{Limitations}

Limitations to this study include the potential for gaps in coverage in certain geographic regions, reflecting limiting our searches to
English-language publications. These gaps may be particularly evident in countries with long histories of vector-borne disease management, such as China and Russia, that have extensive bodies of research not readily accessible due to language barriers in the literature (Ruzek et al. 2019, Zhang et al. 2019). While we included many types of mapping approaches and attempted to describe the range of those approaches, those that we perceived as not quantitative were excluded, as was gray literature such as reports and conference abstracts.

\section{Results}

Our initial database searches returned 12,482 records, which yielded 8,608 unique publications. An overview of the literature screening process, following PRISMA guidelines, is shown in Fig. 1. The final screened dataset comprised 303 studies on tick-borne pathogens with a mapped spatial component published between 1995 and 2020. The full database of screened literature is accessible via github. com/viralemergence/tickmaps. Four out of twenty-seven pathogens of interest did not have any associated mapping studies that met our screening criteria: Omsk hemorrhagic fever virus, sawgrass

Table 2. Bacteria and protozoan parasites included in the study

\begin{tabular}{lll}
\hline Pathogen & Disease & Vectors \\
\hline Anaplasma phagocytophilum & Human Granulocytic Anaplasmosis & Amblyomma spp. \\
& & Ixodes spp. \\
Babesia spp. & Babesiosis & Ixodes spp. \\
Borrelia burgdorferi & Lyme borreliosis & Ixodes spp. \\
Borrelia mayonii & Borrelia mayonii disease/Lyme borreliosis & Ixodes spp. \\
Borrelia miyamotoi & Borrelia miyamotoi disease/hard tick relapsing fever & Ixodes spp. \\
Borrelia spp. & Tick relapsing fever & Ornithodoros spp. \\
Coxiella burnetii & Q fever & Dermacentor spp. \\
& & Hyalomma spp. \\
Ehrlichia chaffeensis & Human Monocytic Ehrlichiosis & Amblyomma spp. \\
& & Dermacentor spp. \\
Ehrlichia spp. & & Ixodes spp. \\
Francisella tularensis & Panola Mountain Ehrlichia & Amblyomma spp. \\
& Tularemia & Amblyomma spp. \\
Rickettsia africae & & Dermacentor spp. \\
Rickettsia conorii & & Ixodes spp. \\
Rickettsia parkeri & African tick bite fever & Not in literature \\
Rickettsia rickettsii & Mediterranean spotted fever & Rhipicephalus spp. \\
& Tidewater spotted fever & Amblyomma spp. \\
& Rocky mountain spotted fever & Amblyomma spp. \\
& & Dermacentor spp.
\end{tabular}

Table 3. Eight types of study methodologies defined in this review

\begin{tabular}{ll}
\hline Type of study & Definition (example) \\
\hline Cluster analysis & $\begin{array}{c}\text { Any type of cluster analysis was used, including SatScan cluster analysis, kernel density hotspot modeling, or similar, } \\
\text { e.g. (15). } \\
\text { A species distribution modeling (SDM) algorithm was applied to point data of occurrences of ticks or tick-borne dis- } \\
\text { ease, and the resulting map was a function of environmental drivers of geographic distributions. } \\
\text { Mapping the extent of ticks or tick-borne disease occurrence, based on a systematic or manual review of historical or } \\
\text { published data and expert opinion, typically expressed with administrative boundaries or zones of suspected risk. } \\
\text { Endemicity mapping }\end{array}$ \\
$\begin{array}{l}\text { Maps which included locations of phylogenetic descriptions-e.g. a pie chart of strain type frequency at a given location. } \\
\text { Spatial data points of information (e.g., the incidence of human cases, presence or absence of vectors), presented on a } \\
\text { Point data }\end{array}$ & $\begin{array}{l}\text { Maps of tick-borne disease prevalence, in humans or other hosts, visualized using raw (unaltered and unmodeled) data. } \\
\text { Maps are generated as predicted functions of prevalence through some sort of quantitative modeling. }\end{array}$ \\
$\begin{array}{l}\text { Prevalence mapping } \\
\text { Prevalence modeling } \\
\text { Risk mapping }\end{array}$ & $\begin{array}{c}\text { Projection of a modeled output (such as linear regression model output) onto a continuous geographic area or region, } \\
\text { intended to communicate the geographic extent and intensity of transmission risk. }\end{array}$ \\
\hline
\end{tabular}


virus, Colorado tick fever virus, and Rickettsia africae (Rickettsiales: Rickettsiaceae). Only nine pathogens had more than ten associated mapping studies: ASF virus, CCHF virus, tick-borne encephalitis virus (TBEV), Borrelia burgdorferi (Johnson et al.; Spirocheatales:

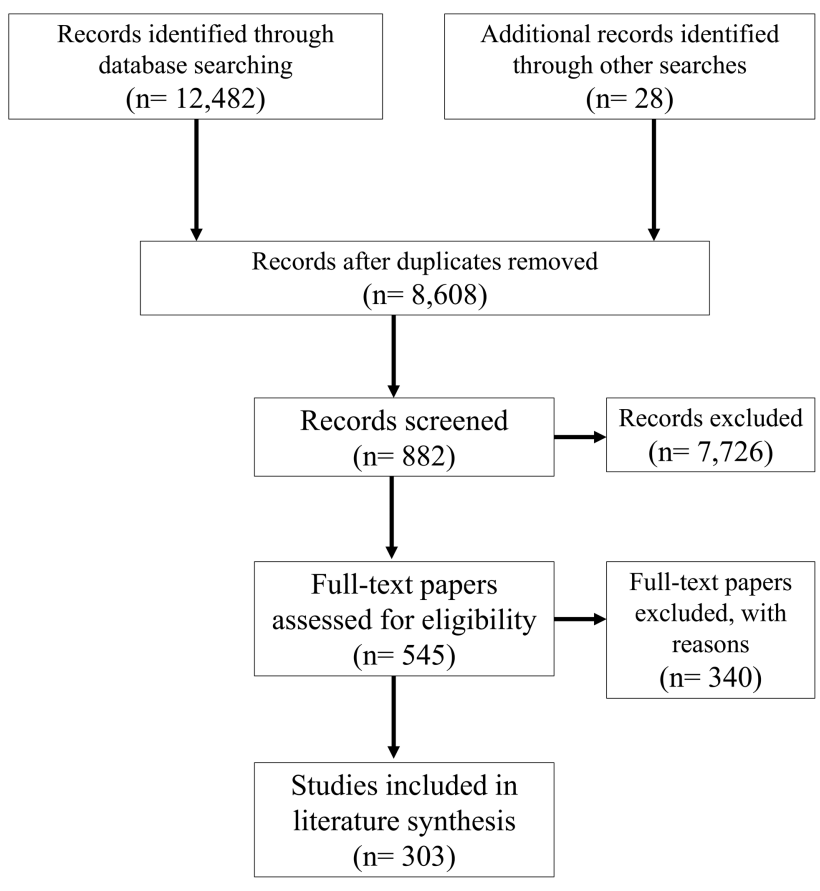

Fig. 1. PRISMA flow diagram outlining the literature search and screening process.
Spirochaetaceae), Anaplasma phagocytophilum (Dumler et al.; Foggie; Rickettsiales: Anaplasmataceae), Coxiella burnetii ((Derrick); Philip; Legionellales: Coxiellaceae), Ehrlichia chaffeensis (Anderson, Dawson and Wilson; Rickettsiales: Anaplasmataceae), Francisella tularensis (Dorofe'ev; McCoy and Chapin; Thiotrichales: Francisellaceae), and Rickettsia rickettsii (Brumpt; Rickettsiales: Rickettsiaceae). While the majority of studies focused on Lyme disease $(40.26 \%)$ or tick-borne encephalitis $(15.51 \%)$, the overall number of published work with a mapping component has increased dramatically across taxa in the past decade (Fig. 2). Tick vectors from seven genera were represented in the final dataset: Amblyomma, Dermacentor, Haemaphysalis, Hyalomma, Ixodes, Ornithodoros, and Rhipicephalus (Fig. 2). Studies with data from Ixodes were the most prevalent, featured in $65.42 \%$ of studies including information on the vector. These typically focused on three species of medical concern: Ixodes scapularis (Say, Ixodida: Ixodidae), Ixodes ricinus (L., Ixodida: Ixodida), Ixodes pacificus (Cooley \& Kohls, Ixodida: Ixodidae).

The eight mapping approaches (Table 3 ) used to classify studies were all represented in the final dataset of screened papers (Fig. 3). Mapping raw occurrence points of pathogens and vectors were the most frequently used approach in communicating spatial information and was used in nearly half $(47.85 \%)$ of screened studies. Risk mapping $(31.02 \%)$ and endemicity mapping $(29.70 \%)$ were also commonly used to communicate the spatial distribution of tick-borne pathogens or the risk of exposure to ticks. Ecological niche modeling was used to estimate distributions, typically for tick vectors, in $22.11 \%$ of studies, and the majority $(76.11 \%)$ of these studies produced niche models with the maximum entropy (MaxEnt) method (Phillips et al. 2006). Cluster analysis was used in $18.48 \%$ of studies, where tests for spatial autocorrelation $(n=15)$

A

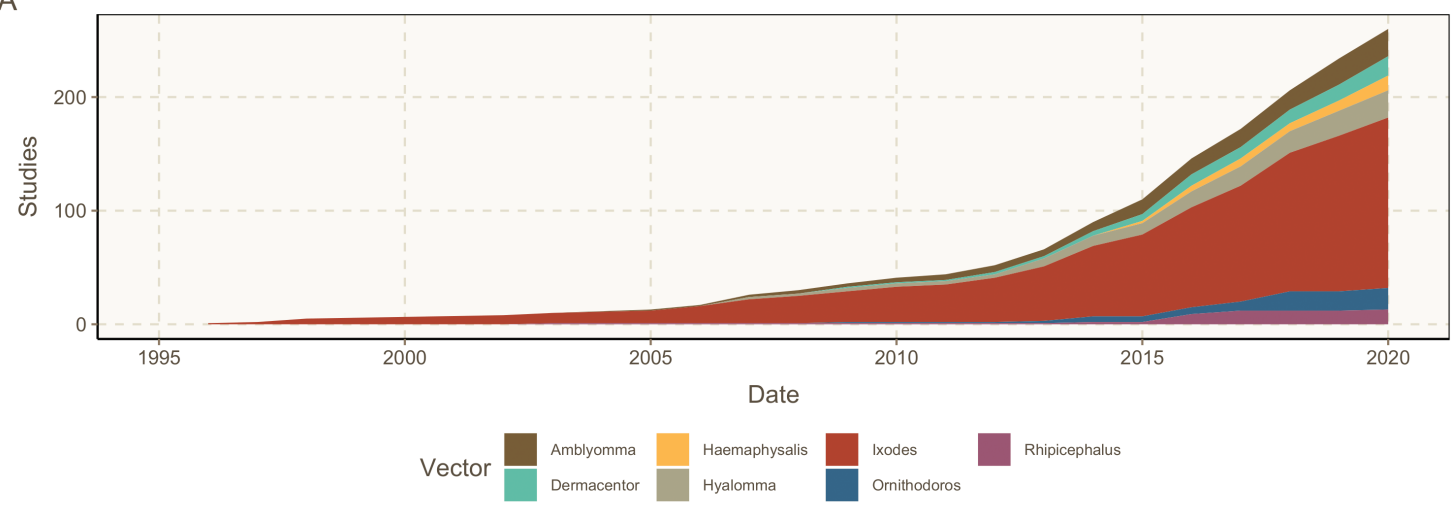

B

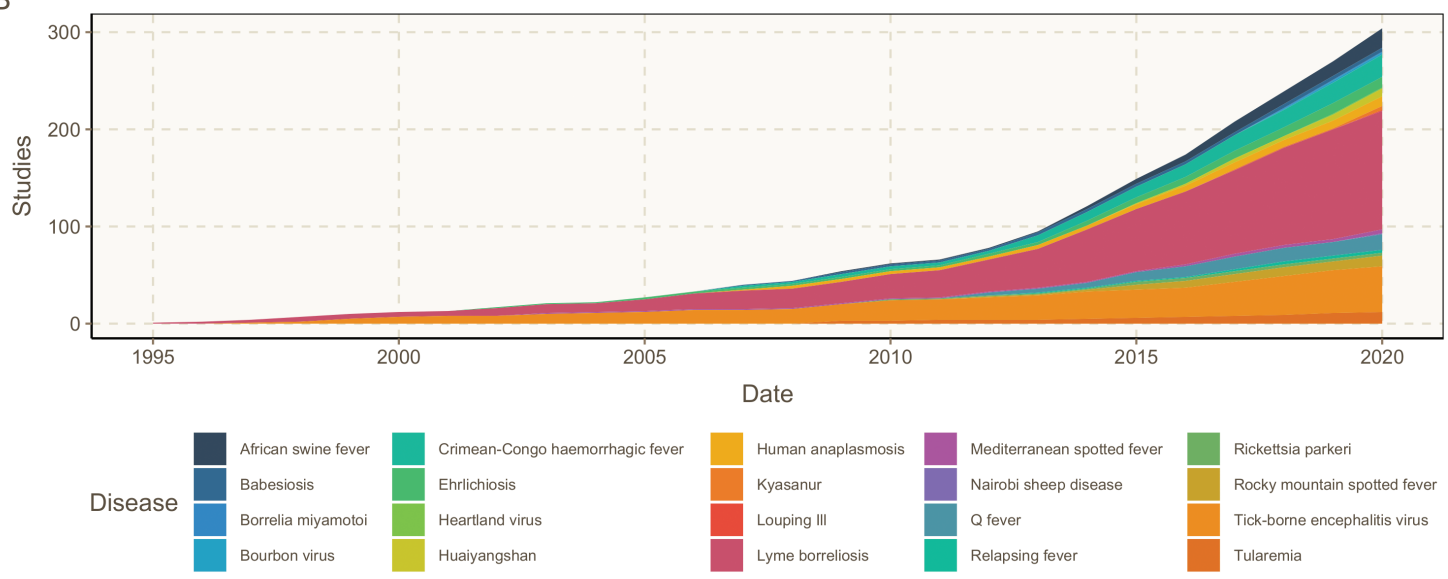

Fig. 2. The cumulative number of studies that collected data about a given genus of tick vector (A) or tick-borne disease (B). 


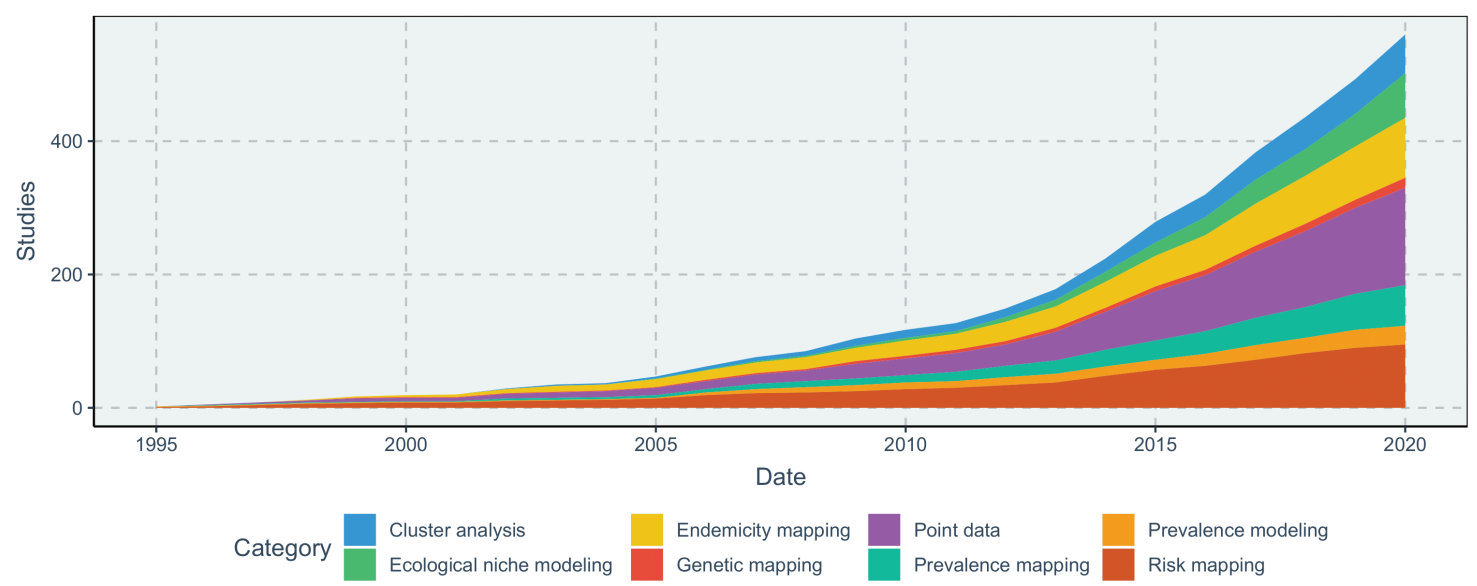

Fig. 3. The cumulative number of studies using any of eight given methodologies.

A

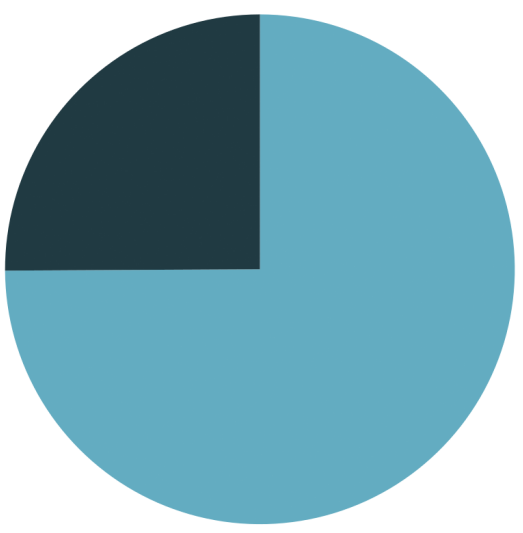

Real pathogen data

Tick data as proxy

C

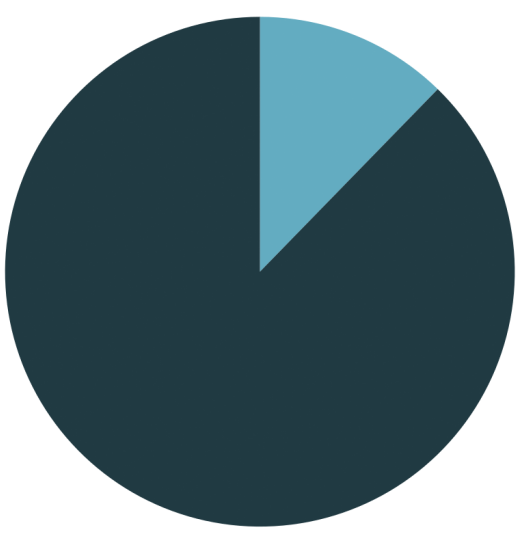

Livestock data

No livestock data
$\mathrm{B}$

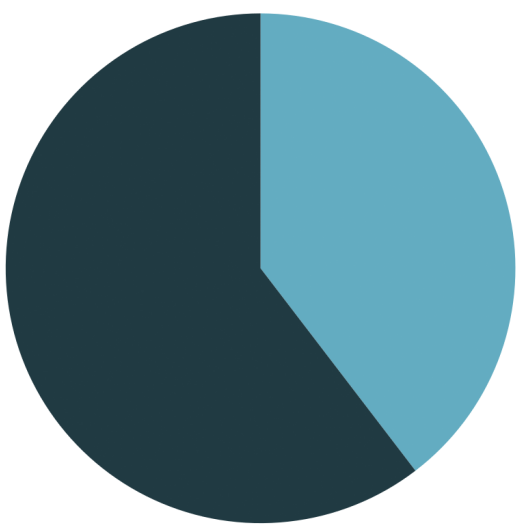

Human case data No human case data

D

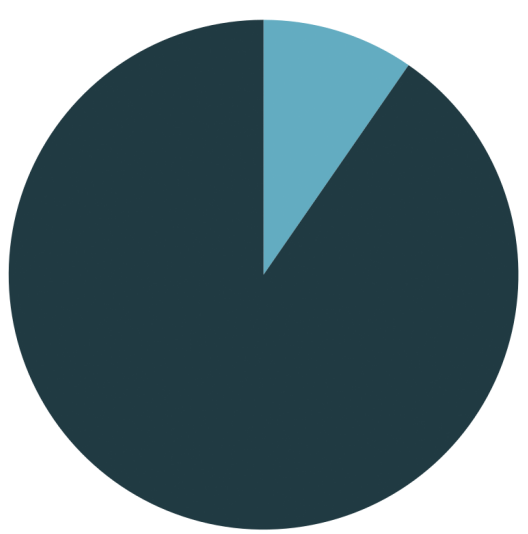

Wildlife data
No wildlife data

Fig. 4. The proportion of studies using different data sources to generate maps of tick-borne disease distribution, transmission, or risk. Many studies use (A) pathogen data directly $(75 \%)$ and (B) human case data $(40 \%)$, while fewer use (C) livestock infection data (12\%) or (D) wildlife infection data (10\%).

and spatial scanning statistics implemented in SaTScan $(n=31)$ were frequently used for cluster detection. Prevalence modeling $(8.58 \%)$ and genetic mapping $(4.62 \%)$ were the methods least used in the final dataset of screened literature.
Data sources used to generate maps varied between studies, where $74.92 \%$ used pathogen records as inputs, and a quarter $(25.08 \%)$ used tick vectors as proxies for the pathogens they transmit (Fig. 4). Previously published datasets were used in 


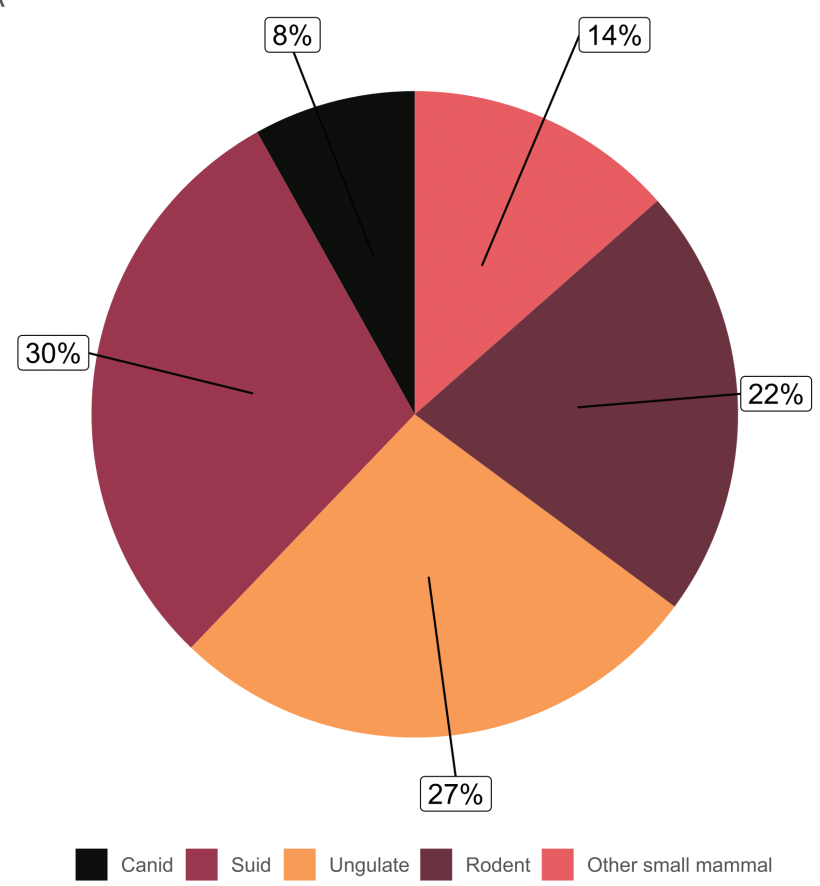

B

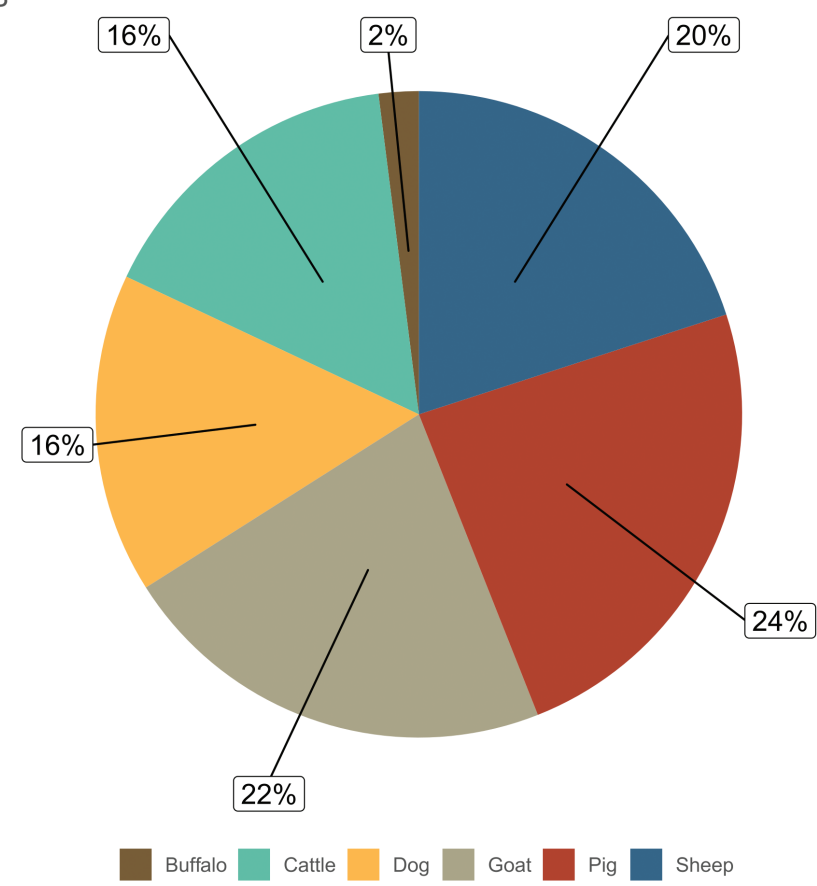

Fig. 5. Proportion of studies with data on different wildlife (A) and livestock species (B).

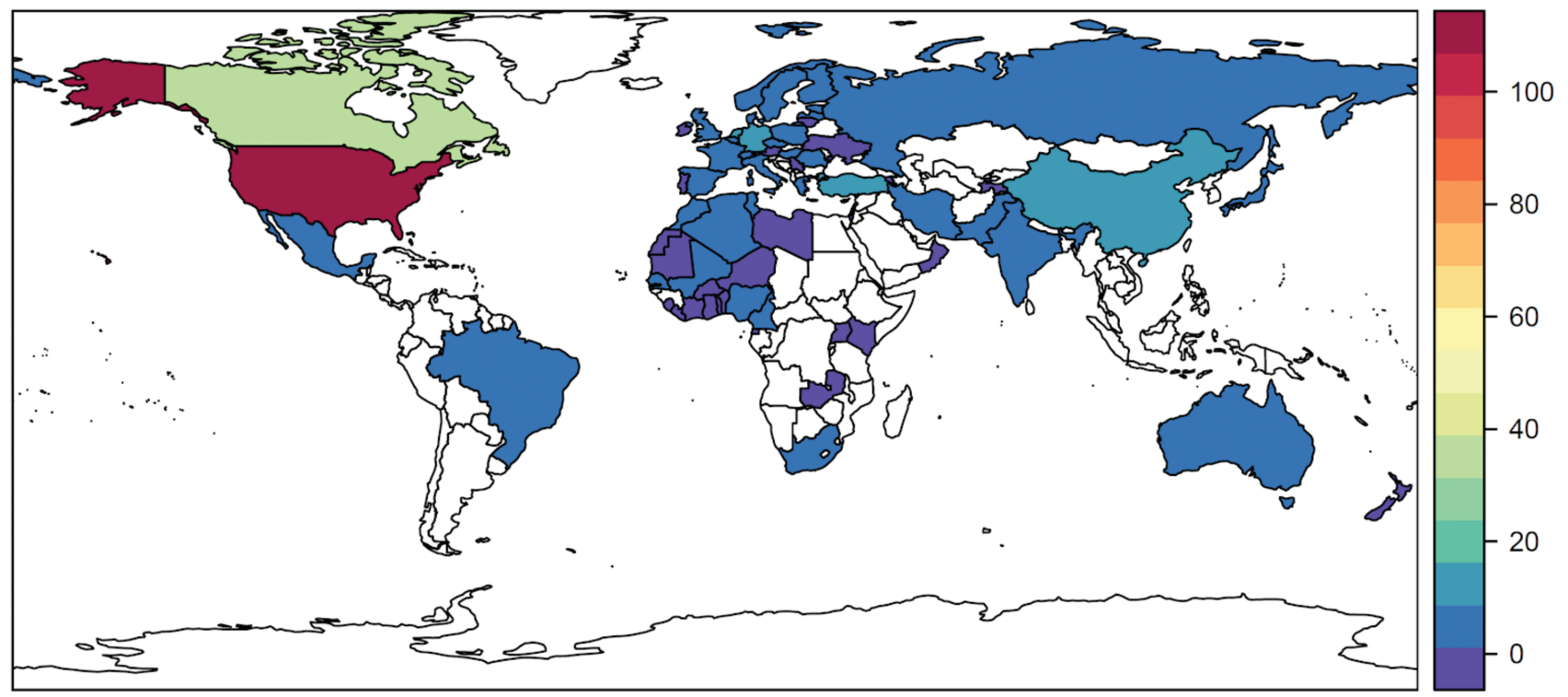

Fig. 6. Number of studies describing the geography of tick-borne disease by country, excluding a handful of explicitly continental studies (most notably 20 in Europe, as well as four in Africa, two in the eastern Mediterranean, one in Asia, and four global mapping studies).

$20.79 \%$ of studies, where resources including museum records, online databases, and literature reviews were commonly leveraged as data sources for spatial analyses. Human cases were used as data inputs in $39.60 \%$ of mapping efforts, and data from other vertebrate hosts such as domesticated livestock $(11.88 \%)$ and wildlife $(9.57 \%)$ were less common (Fig. 4$)$. The relative proportions of domesticated and wildlife hosts sampled for pathogens across studies are presented in Fig. 5. Livestock sampled for pathogens in the literature largely consisted of hoofstock (i.e., cattle, sheep, and pigs) and domestic dogs; rodents, ungulates, and suids were the groups most frequently sampled for pathogens in wildlife serology studies.

The geographic foci and extent of studies included in the final dataset varied considerably, ranging from highly localized areas to mapped outputs with global extent. We found four global mapping studies on tick-borne pathogens, and a number of explicitly continental studies focused on Europe $(n=20)$, Africa $(n=4)$, the eastern Mediterranean $(n=2)$, and Asia $(n=1)$. Regionally, North American locations were heavily represented in the literature, where $35.31 \%$ of studies were conducted in the United States, $11.55 \%$ in Canada, 
and $2.64 \%$ in Mexico (Fig. 6). While global mapping efforts are comparably low compared to North America, there are conspicuous regional gaps in mapped tick-borne disease studies, notably in portions of South America, Africa, the Middle East, Central Asia, and Southeast Asia.

\section{Discussion}

In this study, we performed a systematic review of scientific literature that has mapped tick-borne diseases and quantified our findings in terms of distribution among pathogens, vectors, methods, geographic scope, and other attributes. Together, these provide a reasonable approximation of the current literature's coverage of tick-borne diseases. Our review has demonstrated marked increases in both the number and diversity of work with spatial foci. Still, we have identified gaps in our geographic knowledge of tick-borne diseases. In many instances, basic natural history research to characterize pathogens and vectors will be important to improve the utility of risk mapping for understudied transmission systems. Efforts to expand surveillance of lesser-known pathogens, document sylvatic cycles, and increase the capacity for tick-borne disease surveillance in underrepresented regions will also help support future public health work.

\section{Why Tick-Borne Diseases Are Difficult to Map}

Maps are commonly used to provide a tangible (and graphical) perspective on the "where" of disease risk and can be used as part of the surveillance, prevention, and intervention toolbox in public health. Maps of vector-borne diseases carry an additional layer of complexity, as transmission risk is a combination of the abundance and behavior of vectors, the presence of the disease, and the opportunity for human infection. The data streams to describe vector and pathogen distributions often arise from data collection in historically disparate fields (i.e., entomology and infectious disease epidemiology, respectively). In some instances, key vectors for zoonoses also remain unknown, as is the case for Crimean-Congo hemorrhagic fever virus (Okely et al. 2020), which may have different principal tick vectors by region. Resolving these data barriers and knowledge gaps is one step towards better geospatial studies.

Tick-borne diseases also pose a unique problem, given that nearly all tick-borne viruses are zoonotic, which necessitates a view of their emergence and risk landscapes based on sylvatic and enzootic cycles. For comparison, these transmission cycles are very well researched for some mosquito-borne diseases, such as yellow fever, and knowledge of pathogen-vector-host relationships can be used to improve risk mapping efforts (Jentes et al. 2011). While we have a firm understanding of sylvatic cycles for some tickborne diseases, namely Lyme disease, that operationalized view of transmission does not exist for most tick-borne pathogens, and vertebrate hosts were only considered in a fraction of studies in our literature database. There are many instances where the full components of tick-borne enzootic transmission cycles are either poorly understood or completely unknown (Kemenesi and Bányai 2018, Orkun and Emir 2020). This presents a challenge in accurately mapping distributions of tick-borne diseases, particularly when tick vectors are widespread relative to the pathogens they transmit (Lippi et al. 2020). In these instances, geographic transmission risk and the potential for spillover events would perhaps be better estimated with data on wildlife reservoirs and enzootic transmission patterns. This is a challenging endeavor, however, as establishing enzootic cycles for tick-borne pathogens typically requires extensive data collection in the field and laboratory, often calling for specialized expertise in wildlife sampling that is not typically incorporated into traditional public health surveillance systems (Hamer et al. 2012). Data gaps are again characterized by disciplinary divides, as wildlife disease surveillance usually occurs separately from acarological collections, within-vector pathogen surveillance collection, and human public health records collections. Gaps in our knowledge regarding transmission cycles, therefore, present a major obstacle to quantifying and mapping the risk of exposure.

\section{HowTick-Borne Diseases Are Mapped}

Although we recorded a tremendous diversity of approaches, we found that simple occurrence maps (i.e., displaying raw data points for either pathogens or their vectors) were the most common form of spatial data visualization. Dot maps of disease cases have long been used in epidemiology to communicate basic spatial information, and they remain a frequently used mapping approach that may complement more advanced quantitative methods (Smith et al. 2015). We also found that approximately one-quarter of the studies in this review relied on tick presence as a proxy for pathogen presence and transmission risk. This is an intuitive way to formalize knowledge about the geographic range of risk when pathogen distributions are poorly sampled or unknown. However, maps derived solely from vector data underscore a clear need to refine perceptions of geographic risk through sampling efforts that focus on pathogens.

Mapping studies that employed more analytical approaches for spatial statistics or modeling (such as interpolated risk mapping, ecological niche models, and cluster analysis) have become more common especially in the past five years, likely due to advances in the diversity of modeling algorithms, availability of open-source software, and increasing adoption of these methods in disease ecology. Perhaps most of all, we observed that these approaches relied on the existence of an ecosystem of open, accessible raw data describing the occurrence of ticks, pathogens, and clinical and veterinary cases. This secondary use of data was perhaps most evident in ecological niche modeling studies, where species presence data are commonly used as input for modeling algorithms (Elith and Leathwick 2009). This practice, while pragmatic, comes with the caveat that much work on establishing the spatial risk of tick-borne diseases is hinged on a relatively small pool of existing data. This problem is exacerbated when diseases are rare events under current surveillance practices, or when tick vectors are challenging to sample, such as soft ticks in the genus Ornithodoros (Donaldson et al. 2016). We, therefore, recommend an emphasis on novel data collections, when possible, in future research.

Finally, we noted that despite substantial interest in the expansion of tick-borne diseases over time, there was fairly limited work that conclusively established this pattern. We encountered hundreds of examples of something akin to risk maps for tick-borne diseases, but most are so different in input and mapping technique as to be incomparable across studies, a lack of intercomparability that can stymie attempts to describe change over time. This can be addressed by direct work using a combination of modeling and endemicity mapping to update historical or baseline distributions, and project future areas of vulnerability; like other work, we note that this kind of work is heavily reliant on detailed, real-time primary data.

\section{Which Tick-Borne Diseases Are Mapped}

A small number of tick-borne diseases have been exceptionally wellstudied and well-mapped. We found a preponderance of studies with information about Lyme disease and its vectors. Lyme disease has become the most frequently reported vector-borne disease in the United States, Canada, and Europe, a trend which underlies the geographic 
distribution of the research identified in this study (Lindgren and Jaenson 2006, Shapiro 2014, Lindsay 2016). The prevalence of Lyme disease mapping studies in the literature is unsurprising, as Lyme disease has been previously identified as a major research target, both in disease ecology and public health efforts (Han and Ostfeld 2019, Mac et al. 2019). Similarly, TBEV is also prioritized, likely due to its relative prevalence in humans, long history of its presence as a livestock-related issue, and the intensity of research on this particular disease in Russia (National Academies of Sciences, Engineering, and Medicine et al. 2016, Ruzek et al. 2019, Bojkiewicz et al. 2020). The wealth of existing data for Lyme disease and TBEV, combined with ongoing surveillance efforts, translate into transmission systems that are extensively mapped across spatial and temporal scales, compared to other tick-borne diseases.

With these few exceptions, the majority of tick-borne pathogens are undermapped. Expanded pathogen diversity in mapping studies is mostly relegated to the past decade, a period which coincides with gains in the knowledge of tick-borne pathogen taxonomy, increased awareness of burden, and heightened public health interest (Vayssier-Taussat et al. 2013, Eisen and Eisen 2018, Pollet et al. 2020). Nevertheless, pathogens better represented in mapping studies are typically those that share common vectors with extensively studied pathogens. For example, pathogens that are also transmitted by I. scapularis and I. ricinus (the primary vectors of Lyme borreliosis and TBEV, respectively) tended to be better described in our data, often as part of integrative surveillance focused on these specific vectors instead of anyone pathogen. Pathogens capable of transmission through agricultural production systems were also the focus of many mapping studies, even when the human burden of zoonotic transmission is comparatively low, as is the case with African swine fever and Q fever. In these instances, the bulk of mapped studies stem from the existence of established surveillance in livestock, management of wildlife populations, or testing of agricultural products (e.g., bulk tank milk testing) (Hilbert et al. 2015, Food and Agriculture Organization of the United Nations 2019).

In this review, we find several pathogens of increasing public health importance that would make good candidates for targeted surveillance efforts, where areas of transmission risk are largely derived from vector distributions. For example, four pathogensOmsk hemorrhagic fever virus, sawgrass virus, Colorado tick fever virus, and Rickettsia africae-were entirely absent from the results of our literature searches, and represent important future priorities. Similarly, proportionally understudied pathogens transmitted by I. scapularis-including A. phagocytophilum, E. chaffeensis, and Powassan virus-could be more regularly involved in vector surveillance efforts. In a similar vein, the geographic distribution of reviewed literature indicates several regional disparities in mapped tick-borne disease research, highlighting potential opportunities for increased research efforts. South America, for example, is represented in the tick mapping literature by only two studies conducted in Brazil, despite having confirmed cases of tick-borne diseases and known tick species of medical importance (Guglielmone et al. 2006). Documentation of zoonotic pathogens on the continent is similarly underfunded, and tick-borne transmission cycles are an active area of research (Guglielmone et al. 2006, Rodriguez-Morales et al. 2018). Identifying regional priorities for surveillance based on clinical and veterinary significance, and expanding the purview of tick-borne disease mapping using participatory approaches alongside quantitative and GIS work, will help manage the burden of tick-borne disease where it remains the highest.

\section{Acknowledgments}

CJC and SJR were supported by funding to the Viral Emergence Research Initiative (VERENA) consortium including NSF BII 2021909. CAL, HDG, and SJR were funded by NIH 1R01AI136035-01. ALW and SJR were additionally funded by CDC grant 1U01CK000510-01: Southeastern Regional Center of Excellence in Vector-Borne Diseases: The Gateway Program. This publication was supported by the Cooperative Agreement Number above from the Centers for Disease Control and Prevention. Its contents are solely the responsibility of the authors and do not necessarily represent the official views of the Centers for Disease Control and Prevention.

\section{Author Contributions}

All authors contributed to the conception, design, and writing of the study. CAL and ALW gathered data, and CJC generated data visualizations.

\section{Data Availability}

All data and code are available at github.com/viralemergence/ tickmaps

\section{References Cited}

Brackney, D. E., and P. M. Armstrong. 2016. Transmission and evolution of tick-borne viruses. Curr. Opin. Virol. 21: 67-74.

Bush, L. M., and M. T. Vazquez-Pertejo. 2018. Tick borne illness-Lyme disease. Disease-a-Month. 64: 195-212.

Bojkiewicz, E., K. Toczyłowski, and A. Sulik. 2020. Tick-borne encephalitis - a review of current epidemiology, clinical symptoms, management and prevention. Przegl. Epidemiol. 74: 316-325.

Brown, R. N., R. S. Lane, and D. T. Dennis. 2005. Geographic distributions of tick-borne diseases and their vectors, pp. 363-391. In J. Goodman, D. T. Dennis, and D. Sonenshine (eds.), Tick-borne diseases of humans. ASM Press, Washington, DC.

Carlson, C. J. 2020. Embarcadero: Species distribution modelling with Bayesian additive regression trees in R. Methods Ecol. Evol. 11: 850-858.

CDC. 2018. Tickborne diseases of the United States: A reference manual for health care providers, 5 th ed. Centers for Disease Control and Prevention, Division of Vector-Borne Diseases, Fort Collins, CO.

Dantas-Torres, F., B. B. Chomel, and D. Otranto. 2012. Ticks and tick-borne diseases: a One Health perspective. Trends Parasitol. 10: 437-436.

Donaldson, T. G., A. A. Pèrez de León, A. Y. Li, I. Castro-Arellano, E. Wozniak, W. K. Boyle, R. Hargrove, H. K. Wilder, H. J. Kim, P. D. Teel, et al. 2016. Assessment of the geographic distribution of Ornithodoros turicata (Argasidae): climate variation and host diversity. PLoS Negl. Trop. Dis. 10: e0004383.

Eisen, R. J., and L. Eisen. 2018. The blacklegged tick, Ixodes scapularis: an increasing public health concern. Trends Parasitol. 34: 295-309.

Eisen, R. J., and C. D. Paddock. 2020. Tick and tickborne pathogen surveillance as a public health tool in the United States. J. Med. Entomol. tjaa087. doi:10.1093/jme/tjaa087

Eisen, L., and K. C. Stafford. 2020. Barriers to effective tick management and tick-bite prevention in the United States (Acari: Ixodidae). J. Med. Entomol. tjaa079. doi:10.1093/jme/tjaa079

Elith, J., and J. R. Leathwick. 2009. Species distribution models: ecological explanation and prediction across space and time. Annu. Rev. Ecol. Evol. Syst. 40: 677-697.

Espinaze, M. P. A., E. Hellard, I. G. Horak, and G. S. Cumming. 2018. Domestic mammals facilitate tick-borne pathogen transmission networks in South African wildlife. Biol. Conserv. 221: 228-236.

Fatmi, S. S., R. Zehra, and D. O. Carpenter. 2017. Powassan Virus-A New Reemerging Tick-Borne Disease. Front. Public Health. 5: 342. 
Food and Agriculture Organization of the United Nations. 2019. African swine fever in wild boar: Ecology and biosecurity. Food \& Agriculture Org., Rome, Italy.

Grace, D., J. Lindahl, F. Wanyoike, B. Bett, T. Randolph, and K. M. Rich. 2017. Poor livestock keepers: ecosystem-poverty-health interactions. Philos. Trans. R. Soc. Lond. B Biol. Sci. 372: 20160166.

Guglielmone, A. A., L. Beati, D. M. Barros-Battesti, M. B. Labruna, S. Nava, J. M. Venzal, A. J. Mangold, M. P. J. Szabó, J. R. Martins, D. GonzálezAcuña, et al. 2006. Ticks (Ixodidae) on humans in South America. Exp. Appl. Acarol. 40: 83-100.

Han, B. A., and R. S. Ostfeld. 2019. Topic modeling of major research themes in disease ecology of mammals. J. Mammal. 100: 1008-1018.

Hamer, S. A., G. J. Hickling, R. Keith, J. L. Sidge, E. D. Walker, and J. I. Tsao. 2012. Associations of passerine birds, rabbits, and ticks with Borrelia miyamotoi and Borrelia andersonii in Michigan, U.S.A. Parasit. Vect. 5: 231

Hilbert, A., T. Andres, R. Werner, R. Wehr, A. Fröhlich, F. J. Conraths, and K. Henning. 2015. Detection of Coxiella burnetii in dairy cattle bulk tank milk and single tank milk samples by confirmatory testing. Berl. Munch. Tierarztl. Wochenschr. 128: 271-277.

Jentes, E. S., G. Poumerol, M. D. Gershman, D. R. Hill, J. Lemarchand, R. F. Lewis, J. E. Staples, O. Tomori, A. Wilder-Smith, and T. P. Monath; Informal WHO Working Group on Geographic Risk for Yellow Fever. 2011. The revised global yellow fever risk map and recommendations for vaccination, 2010: consensus of the Informal WHO Working Group on Geographic Risk for Yellow Fever. Lancet. Infect. Dis. 11: 622-632.

Kitron, U. 1998. Landscape epidemiology and epidemiology of vector-borne diseases: tools for spatial analysis. J. Med. Entomol. 35:435-45.

Kuris, A. M. 2012. The global burden of human parasites: who and where are they? How are they transmitted? J. Parasitol. 98: 1056-1064.

Kemenesi, G. and K. Bányai. 2018. Tick-borne flaviviruses, with a focus on powassan virus. Clin. Microbiol. Rev. 32: e00106-17.

Lindsay, L. R. 2016. Present state of common vector-borne diseases in Canada. Can. Commun. Dis. Rep. 42: 200-201.

Lindgren, E., and T. G. T. Jaenson. 2006. Lyme borreliosis in Europe: Influences of climate and climate change, epidemiology, ecology and adaptation measures (No. EUR/04/5046250). World Health Organization Europe, Copenhagen, Denmark.

Liberati, A., D. G. Altman, J. Tetzlaff, C. Mulrow, P. C. Gøtzsche, J. P. Ioannidis, M. Clarke, P. J. Devereaux, J. Kleijnen, and D. Moher. 2009. The PRISMA statement for reporting systematic reviews and meta-analyses of studies that evaluate health care interventions: explanation and elaboration. J. Clin. Epidemiol. 62: e1-34.

Lippi, C. A., H. D. Gaff, A. L. White, H. K. St. John, A. L. Richards, and S. J. Ryan. 2020. Exploring the Niche of Rickettsia montanensis (Rickettsiales: Rickettsiaceae) infection of the american dog tick (Acari: Ixodidae), using multiple species distribution model approaches. J. Med. Entomol. tjaa263. doi:10.1093/jme/tjaa263

Moher, D. 2009. Preferred reporting items for systematic reviews and metaanalyses: The PRISMA statement. Ann. Inter. Med. 151: 264-269.

Mac, S., S. R. da Silva, and B. Sander. 2019. The economic burden of Lyme disease and the cost-effectiveness of Lyme disease interventions: a scoping review. PLoS One. 14: e0210280.

National Academies of Sciences, Engineering, and Medicine, Health and Medicine Division, Board on Global Health, and Forum on Microbial Threats. 2016. Global health impacts of vector-borne diseases: Workshop summary. National Academies Press, Washington, D.C.

Orkun, O. and H. Emir. 2020. Identification of tick-borne pathogens in ticks collected from wild animals in Turkey. Parasitol. Res. 119: 3083-3091.
Okely, M., R. Anan, S. Gad-Allah, and A. M. Samy. 2020. Mapping the environmental suitability of etiological agent and tick vectors of CrimeanCongo hemorrhagic fever. Acta Trop. 203: 105319.

ProMED. 2021. ProMED-mail. Program for Monitoring Emerging Diseases, International Society for Infectious Diseases. https://promedmail.org/.

Phillips, S. J., R. P. Anderson, and R. E. Schapire. 2006. Maximum entropy modeling of species geographic distributions. Ecol. Modell. 190: 231-259.

Pollet, T., H. Sprong, E. Lejal, A. I. Krawczyk, S. Moutailler, J. F. Cosson, M. Vayssier-Taussat, and A. Estrada-Peña. 2020. The scale affects our view on the identification and distribution of microbial communities in ticks. Parasit. Vectors. 13: 36

Reeves, W. K., A. D. Loftis, W. L. Nicholson, and A. G. Czarkowski. 2008. The first report of human illness associated with the Panola Mountain Ehrlichia species: a case report. J. Med. Case Rep. 2: 139.

Rochlin, I., D. V. Ninivaggi, and J. L. Benach. 2019. Malaria and Lyme disease - the largest vector-borne US epidemics in the last 100 years: success and failure of public health. BMC Public Health. 19: 804.

Rodriguez-Morales, A. J., D. K. Bonilla-Aldana, S. E. Idarraga-Bedoya, J. J. Garcia-Bustos, J. A. Cardona-Ospina, and Á. A. Faccini-Martínez. 2018. Epidemiology of zoonotic tick-borne diseases in Latin America: are we just seeing the tip of the iceberg? F1000Res. 7: 1988.

Ruzek, D., T. Avšič Županc, J. Borde, A. Chrdle, L. Eyer, G. Karganova, I. Kholodilov, N. Knap, L. Kozlovskaya, A. Matveev, et al. 2019. Tickborne encephalitis in Europe and Russia: Review of pathogenesis, clinical features, therapy, and vaccines. Antiviral Res. 164: 23-51.

Schiffman, E. K., C. McLaughlin, J. A. E. Ray, M. M. Kemperman, A. F. Hinckley, H. G. Friedlander, and D. F. Neitzel. 2016. Underreporting of lyme and other tick-borne diseases in residents of a high-incidence county, minnesota, 2009. Zoonoses Public Health. 65: 230-237.

Shapiro, E. D. 2014. Clinical practice. Lyme disease. N. Engl. J. Med. 370: 1724-1731.

Schluth, C. G., C. J. Standley, S. Bansal, and C. J. Carlson. 2020. Mapping the human helminthiases: advances and gaps in neglected disease surveillance. MedRxiv. doi:10.1101/2020.10.30.20223529

Smith, K. F., M. Goldberg, S. Rosenthal, L. Carlson, J. Chen, C. Chen, and S. Ramachandran. 2014. Global rise in human infectious disease outbreaks. J. R. Soc. Interface. 11(101): 20140950.

Smith, C. M., S. C. Le Comber, H. Fry, M. Bull, S. Leach, and A. C. Hayward. 2015. Spatial methods for infectious disease outbreak investigations: systematic literature review. Euro Surveill. 20.

Tick-Borne Disease Working Group. 2018. 2018 Report to Congress. U.S. Department of Health and Human Services, Washington, D.C.

Torgerson, P. R., and C. N. Macpherson. 2011. The socioeconomic burden of parasitic zoonoses: global trends. Vet. Parasitol. 182: 79-95.

Vayssier-Taussat, M., S. Moutailler, L. Michelet, E. Devillers, S. Bonnet, J. Cheval, C. Hébert, and M. Eloit. 2013. Next generation sequencing uncovers unexpected bacterial pathogens in ticks in western Europe. PLos One. 8: e81439.

WHO. 2021. Disease Outbreak News. World Health Organization, Geneva, Switzerland. https://www.who.int/csr/don/en/.

White, A., and H. Gaff. 2018. Review: Application of Tick Control Technologies for Blacklegged, Lone Star, and American Dog Ticks. J. Integr. Pest Manag. 9: 1-10.

Woolhouse, M. E., R. Howey, E. Gaunt, L. Reilly, M. Chase-Topping, and N. Savill. 2008. Temporal trends in the discovery of human viruses. Proc. Biol. Sci. 275: 2111-2115.

Zhang, G., D. Zheng, Y. Tian, and S. Li. 2019. A dataset of distribution and diversity of ticks in China. SciData. 6: 105 